\title{
Targeting proinsulin to local immune cells using an intradermal microneedle delivery system; a potential antigen- specific immunotherapy for type 1 diabetes
}

Farah Arikat ${ }^{a}$, Stephanie J. Hanna ${ }^{\text {b }}$, Ravinder K. Singh ${ }^{\text {b}}$, Luciano Vilela ${ }^{c}$, F. Susan Wong ${ }^{b}$, Colin M. Dayan ${ }^{b}$, ${ }^{*}$ Sion A. Coulman ${ }^{a}$, James C. Birchall ${ }^{a}$

${ }^{a}$ School of Pharmacy and Pharmaceutical Sciences, Cardiff University, Cardiff, CF10 3NB, UK.

${ }^{\mathrm{b}}$ School of Medicine, Cardiff University, Cardiff, CF14 4XN, UK.

${ }^{\mathrm{c}}$ Biomm S.A., Nova Lima -MG, 34019-000, Brazil.

${ }^{*}$ Corresponding author.

Email address: CoulmanSA@cardiff.ac.uk (S.A. Coulman)

Declarations of interest: none

\begin{abstract}
Antigen-specific immunotherapy (ASI) has been proposed as an alternative treatment strategy for type 1 diabetes (T1D). ASI aims to induce a regulatory, rather than stimulatory, immune response in order to reduce, or prevent, autoimmune mediated $\beta$-cell destruction, thus preserving endogenous insulin production. The abundance of immunocompetent antigen presenting cells (APCs) within the skin makes this organ an attractive target for immunotherapies. Microneedles (MNs) have been proposed as a suitable drug delivery system to facilitate intradermal delivery of autoantigens in a minimally invasive manner. However, studies to date have employed single peptide autoantigens, which would restrict ASI to patients expressing specific Human Leukocyte Antigen (HLA) molecules, thus stratifying the patient population. This study aims to develop, for the first time, an intradermal MN delivery system to target proinsulin, a large multi-epitope protein capable of inducing tolerance in a heterogenous (in terms of HLA status) population of T1D patients, to the immunocompetent cells of the skin. An optimized three component coating formulation containing proinsulin, a diluent and a surfactant, facilitated uniform and reproducible coating of $>30 \mu \mathrm{g}$ of the active pharmaceutical ingredient on a stainless steel MN array consisting of thirty $500 \mu \mathrm{m}$ projections. When applied to a murine
\end{abstract}


model these proinsulin-coated MNs efficiently punctured the skin and after a limited insertion time (150 seconds) a significant proportion of the therapeutic payload (86\%) was reproducibly delivered into the local tissue. Localized delivery of proinsulin in non-obese diabetic (NOD) mice using the coated MN system stimulated significantly greater proliferation of adoptively transferred antigen-specific CD8+ T cells in the skin draining lymph nodes compared to a conventional intradermal injection. This provides evidence of targeted delivery of the multi-epitope proinsulin antigen to skinresident APCs, in vivo, in a form that enables antigen presentation to antigen-specific $T$ cells in the local lymph nodes. The development of an innovative coated MN system for highly targeted and reproducible delivery of proinsulin to local immune cells warrants further evaluation to determine translation to a tolerogenic clinical outcome.

Keywords: Microneedle, diabetes, tolerance, skin, immunotherapy, proinsulin

\section{Introduction}

Type 1 diabetes (T1D) is an autoimmune disorder that causes hyperglycemia as a result of $T$ cell-mediated destruction of the insulin-producing pancreatic $\beta$-cells (Burrack et al. 2017). Insulin-replacement provides symptomatic treatment but this relies on repeated daily injections in a complex dosing regimen that must be revised regularly in an attempt to mimic the refined glucose control that is afforded by a functional biological system. Treatment is a lifelong commitment for the patient and glucose control is often sub-optimal, resulting in significant health-related complications (Smith and Peakman 2018). Insulin is therefore not a cure for T1D (Pathiraja et al. 2015), i.e. it does not halt the autoimmune process or restore $\beta$-cell function to enable endogenous insulin production and glucose control (Chen et al. 2017). Antigen-specific immunotherapy (ASI) is an emerging T1D treatment strategy that aims to induce a regulatory response to the autoantigen to lessen, or even prevent, $\beta$-cell destruction (Smith and Peakman 2018) and thus preserve endogenous insulin production. However, there is a delicate balance between the regulatory and stimulatory immune pathways (Arce-Sillas et al. 2016) and therefore the nature of the autoantigen, its dose and the route of delivery need to be carefully tailored (Kroger et al. 2018) to produce a tolerogenic rather than inflammatory response.

The skin is an attractive target organ for ASI due to the abundance of antigen presenting cells (APCs) in both the epidermis and dermis, i.e. Langerhans cells (LCs) 
and dermal dendritic cells (dDCs) respectively (Ho and Kupper 2019). These cells are able to capture autoantigens within the local environment, migrate to the draining lymph nodes (LNs) and present them to antigen-specific T cells (Leone et al. 2017), which direct and orchestrate the subsequent immune response. Whilst a conventional needle and syringe can be used to deliver such antigens into the skin, the millimeter dimensions of the needle cannot exclusively target the most APCabundant superficial layers of the tissue. Delivery of a liquid bolus into the skin, from either a conventional injection or a hollow microneedle, also causes physical expansion of the tissue (commonly described as bleb formation), which leads to significant architectural disruption in the dermis and/or at the epidermal-dermal barrier (Gupta et al. 2011; Dul et al. 2017), local infiltration by inflammatory cells (Liu et al. 2003; Liu et al. 2005) and release of the pro-inflammatory cytokine, TNF-a (Ali et al. 2015). Less inflammatory and targeted methods of intradermal administration are therefore highly desirable for therapies that aim to promote tolerization, i.e. ASI.

Microneedle (MN) technology provides a minimally invasive means to overcome the principal skin barrier (the stratum corneum) for local delivery of high molecular weight (bio)therapeutics such as cyclosporin A (Jeong et al. 2018), glucagon (PalylykColwel and Ford 2017), parathyroid hormone (1-34) (Naito et al. 2018) and insulin (Yu et al. 2015; Lau et al. 2017). This is achieved without causing the pain and/or bleeding that is associated with conventional intradermal injection techniques (Haq et al. 2009; Arya et al. 2017). This is particularly attractive for ASI, where minimising tissue trauma upon delivery of an autoantigen may reduce the maturation of local epidermal and dermal dendritic cells and promote activation of regulatory rather than effector T cells (Petzold et al. 2010; Dalod et al. 2014; Iberg et al. 2017), i.e. a protolerogenic response. Coated $\mathrm{MNs}$ are a sub-category of MNs that facilitate intradermal delivery of therapeutics by dissolution of the coating formulation within the local biological environment following their application to the skin surface (Prausnitz 2017). This MN sub-type targets LCs and dDCs (Turvey et al. 2019) but minimizes damage to the local tissue and therefore may be particularly useful in the clinical context of ASI.

Previous efforts to produce a coated MN delivery system for ASI of T1D have resulted in development of formulations for a single peptide autoantigen (Zhao et al. 2016). However, single epitope peptides are only clinically relevant in patients that possess the complementary Human Leukocyte Antigen (HLA) molecules on the surface of their APCs. Therefore, the clinical potential of a single peptide autoantigen 
is restricted to sub-populations of patients with a specific HLA status (So et al. 2018). For example, only those individuals who express an HLA genotype that includes HLA-DR4, which is expressed in 30-50\% of people with T1D (Steck and Rewers 2011), could potentially benefit from ASI using the C19-A3 autoantigen (Alhadj Ali et al. 2017). Proinsulin (PI), the 86-amino acid precursor of insulin (Arai et al. 2018), is a major autoantigen implicated in T1D development in both humans and mice (Di Lorenzo et al. 2007) with $\mathrm{PI}$ and $\mathrm{PI}$ peptide- reactive $\mathrm{T}$ cells having been previously isolated from the T1D mouse model (Wegmann et al. 1994; Wong et al. 1996) and more recently from human subjects with T1D (Michels et al. 2017; Kent et al. 2017). Single PI peptides have been previously studied as ASI for T1D with more recent peptides including insulin B9-23 (Zhao et al. 2016), C19-A3 (Alhadj Ali et al. 2017) and insulin B22-C12 (De Groot et al. 2019). The PI C-peptide is a 31-amino acid peptide which is excised from PI to generate insulin and it was reported that the CD4+ $T$ cell response to C-peptide in the peripheral blood of patients with recent onset T1D was significantly greater than that of healthy controls (So et al. 2018), indicating the importance of this antigen in T1D. The full-length C-peptide was found to be a stronger agonist for C-peptide- specific CD4+ T cell clones than 18-amino acid peptides which incorporated the cognate antigen, eluding to the importance of the presence of multiple epitopes (So et al. 2018). An innovative method of ASI has been recently reported which utilizes the insulin protein as the candidate antigen (Leon et al. 2019). Soluble antigen array insulin $\left(\mathrm{SAgA}_{\mathrm{Ins}}\right)$ consist of multiple insulin molecules conjugated to hyaluronic acid and drain from the site of injection to secondary lymphoid organs. In vitro studies showed that $\mathrm{SAgA}_{\operatorname{lns}}$ bind specifically to insulin-reactive $B$ cells, cause decreased expression of $B$ cell receptors (BCRs) and cause desensitization of the BCR to additional stimulation. This induction of refractory $B C R$ signalling presents a mechanism of peripheral tolerance similar to $B$ cell anergy (Leon et al. 2019).

The complete PI protein contains more epitopes than the aforementioned antigens and therefore provides a potential therapeutic candidate with broader clinical utility, i.e. it would be suitable for a majority rather than a minority of T1D patients. Generating tolerance to multiple epitopes rather than a single autoantigen also has potential advantages in terms of efficacy. In a previous study using multiple peptides from the PI molecule, higher numbers of regulatory T cells in the LNs and less serum PI autoantibodies were generated compared with the use of a single PI peptide (Gibson et al. 2015). This is supported by in vivo studies that have used intraperitoneal injections to demonstrate the potential clinical utility of PI as ASI for 
T1D (Chen et al. 2001; Tian et al. 2014; Yeste et al. 2016). PI, therefore, has significant therapeutic potential for ASI of T1D but has not previously been delivered via the intradermal route, using MNs.

Whilst previous studies for ASI of T1D using MNs have used single peptides (Zhao et al. 2016; Dul et al. 2019), this study aimed to formulate a novel complete PI proteincoated MN system that is able to deliver the PI protein into the superficial layers of the skin, in order to stimulate a localized immune response. Multiple epitopes in the complete protein provide the potential in ASI to generate tolerance to a range of epitopes simultaneously and be therapeutically applicable to more patients. The utilisation of MNs provides a targeted, minimally invasive delivery method causing minimal trauma and local inflammation; beneficial for use in ASI when aiming to generate tolerance. We hypothesize that $\mathrm{PI}$-coated MNs will target immature steadystate dendritic cells (DCs) and/or epidermal LCs that display tolerogenic functions (Steinman et al. 2003; Shklovskaya et al. 2011). Localized delivery of the therapeutic in a solid state may also reduce clearance from the local skin environment and prolong presentation of the antigen in the draining LNs (Zhao et al. 2016), thus promoting sustained exposure of APCs to low doses of the autoantigen. Repeated low doses of antigen to APCs have been shown to induce peripheral tolerance (Steinman et al. 2003) and generate a pro-tolerogenic immune response (Akbarpour et al. 2015; Zhao et al. 2016).

\section{Materials and methods}

\subsection{Reagents and equipment}

Reagents and laboratory consumables were purchased from Fisher Scientific, UK, Sigma-Aldrich, UK, Life Technologies, UK and Becton Dickinson, UK, unless otherwise stated. Cell isolation equipment and reagents were purchased from MACS Miltenyi Biotec, UK. The PI used in these studies was a recombinant human $\mathrm{PI}$ and was a kind gift from Luciano Vilela, Biomm S.A., Brazil. The recombinant human PI has the same structure and amino acid sequence as that of the native human PI, but also incorporates a histidine affinity tag (sequence from N' to C': MAHHHHHHMGR), resulting in a total of 97 amino acids (supplementary figure 1) and a molecular weight of approximately $12,500 \mathrm{~g} / \mathrm{mol}$ (personal communication Luciano Vilela).

\subsection{In vivo model}


The non-obese diabetic (NOD) mouse is the T1D mouse model. NOD/Caj mice were originally obtained from Yale University. G9Ca-/- NOD transgenic mice express CD8 ${ }^{+}$ T cells with the T cell receptor (TCR) of the diabetogenic G9C8 T cell clone (Wong et al. 2009), which recognizes the insulin B15-23 peptide (B15-23), a peptide of the insulin B chain (LYLVCGERG) (Wong et al. 1999). All mice were bred and maintained in specific pathogen-free isolators and ScanTainers at Cardiff University, UK. All animal experiments were approved by Cardiff University ethical review process and conducted under UK Home Office licence in accordance with the UK Animals (Scientific Procedures) Act, 1986 and associated guidelines.

\subsection{Preparation of Pl-coated MNs}

Laser cut medical grade stainless steel MNs were manufactured by Tech-Etch (USA) and consisted of a row of 10 needles, designed to be $500 \pm 50 \mu \mathrm{m}$ long and $200 \mu \mathrm{m}$ wide (base). To prepare the PI MN coating formulation, the solvent, $0.2 \mathrm{M}$ acetic acid (AA) $/ 0.5 \%$ polysorbate 80 , was added to an aliquot of PI powder in a ratio of $100 \mathrm{mg}$ of powder to $1 \mathrm{ml}$ of solvent, resulting in a concentration of $82.9 \pm 8.0 \mathrm{mg} / \mathrm{ml}(\mathrm{n}=58)$ with a $\mathrm{pH}$ of 4.5 . The coating formulation was vortexed intermittently for 2-3 minutes, until clear of aggregates. MN coating was performed as described previously (Zhao et al. 2017). Briefly, $0.5 \mu$ of the coating formulation was loaded into a $10 \mu l$ extended length pipette tip, which was then carefully released from the pipette and used to apply the coating formulation to the MNs. Thirty individual MNs (3 rows of 10 needles) were sequentially coated from a single pipette tip using a manual process that was observed using a Leica ZOOM 2000 illuminated stereomicroscope (Leica Microsystems, Germany). To facilitate topical application, the three rows of MNs were secured in a bespoke MN holder (Cardiff University School of Engineering, UK), as illustrated previously by Zhao et al. (2016).

\subsection{Quantifying the dose of PI coated on MNs}

Each array of $30 \mathrm{MNs}$ was coated in $0.5 \mu \mathrm{l}$ of a formulation containing $82.9 \mathrm{mg} / \mathrm{ml} \mathrm{PI}$ in $0.2 \mathrm{M} \mathrm{AA} / 0.5 \%$ polysorbate 80 . To recover the coated dose from the $\mathrm{MN}$ surface for quantification, Pl-coated MNs were incubated in $500 \mu \mathrm{l}$ of $0.2 \mathrm{M}$ AA for 10 minutes in a $2.75 \mathrm{~L}$ ultrasonic bath $(600 \mathrm{~W})$. The residual formulation in the pipette tips used to coat the MNs were also recovered using this method; these pipette tips were incubated in $200 \mu \mathrm{l}$ of $0.2 \mathrm{M}$ AA and sonicated in the same way. Control solutions were prepared by adding $0.5 \mu \mathrm{l}$ of $\mathrm{PI}$ coating formulation to $499.5 \mu \mathrm{l}$ of $0.2 \mathrm{M} \mathrm{AA}$. Quantification of PI was conducted using the Thermo Scientific Dionex UltiMate 3000 UHPLC. Samples were analyzed on the Ascentis ${ }^{\circledR}$ Express Peptide ES-C18, 2.7 
micron Reversed-Phase HPLC column (10cmx4.6mm) (Sigma-Aldrich, UK). HPLC grade reagents were used to prepare the mobile phases, acetonitrile/0.1\% trifluoroacetic acid (TFA) (mobile phase A) and water/0.1\%TFA (mobile phase B). The HPLC method used a wavelength of $212 \mathrm{~nm}$, a flow rate of $1 \mathrm{ml} / \mathrm{min}$, a $20 \mu \mathrm{l}$ injection volume, a column temperature of $35^{\circ} \mathrm{C}$ and a run time of 8 minutes. A $300 \mu \mathrm{l}$ volume of samples was loaded into $300 \mu \mathrm{HPLC}$ vials and triplicate absorbance readings were obtained for each sample. Peaks were manually integrated and concentrations of samples were interpolated from a standard curve to determine both the mass of PI present on the coated MNs and the residual mass of PI in the pipette tip.

\subsection{Puncture performance of $\mathrm{PI}$-coated $\mathrm{MNs}$ in mouse skin}

Mice were sacrificed by approved methods and frozen for later use. Frozen mice were defrosted and the fur on the scruff of the neck was shaved using electric hair trimmers. The skin was pinched and an array of $30 \mathrm{PI}$-coated MNs, arranged in the MN holder device, was applied to the skin for 2.5 minutes, while a supportive substrate was placed behind the pinched skin (Fig. 1). On removal of the MNs, puncture holes were stained with $1 \%$ methylene blue and counted. Presence of a methylene blue-stained dot signified successful puncture of the skin. Images of the MN-treated skin were acquired using a Nikon D5000 12.3MP Digital SLR Camera (Nikon, UK). This was conducted on a total of 6 mice.

\subsection{Characterising PI delivery from PI-coated $\mathrm{MNs}$ in vivo}

NOD mice were anaesthetized using isoflurane and the fur on the scruff of the neck was shaved with electric hair trimmers. Arrays of $30 \mathrm{PI}$-coated MNs were applied to the skin on the scruff of the neck of the anaesthetized mice for 1, 2.5 or 10 minutes, as described in section 2.5. MNs were then washed to recover residual coated PI, which was quantified as described in section 2.4. Mass balance i.e. the difference between the coated dose of $\mathrm{PI}$ and the dose remaining on the MNs following application, was used to infer the delivered dose. A total of 15 mice were used in this study; 5 mice for each MN application duration investigated. In subsequent studies, coated and non-coated MNs were also inspected by SEM before and after a 2.5 minute application to mouse skin. This was designed to provide qualitative information on the extent and location of PI-coating on MNs both before and after use. MNs were mounted on carbon adhesive discs on aluminium stubs (both from Agar Scientific, UK), placed in the FEI XL-30 FEG environmental scanning electron 
microscope (FEI, USA) and imaged by secondary electron imaging using $10.0 \mathrm{kV}$ beam energy and a working distance of $10 \mathrm{~mm}$.

2.7 Determining if $\mathrm{MN}$ administered $\mathrm{PI}$ is trafficked to $\mathrm{T}$ cells in the draining LNs, in vivo

\subsubsection{PI delivery in vivo}

Arrays of PI-coated MNs were applied to the shaved skin on the scruff of the neck of anaesthetized NOD female mice (6-7 weeks old), as described in section 2.5. An estimated dose of $28.9 \mu \mathrm{g}$ of $\mathrm{PI}$ was delivered to the mouse, based on the intradermal PI deposition results (section 3.3). A conventional ID injection, using a 29G hypodermic needle, was used in the positive control group $(28.9 \mu \mathrm{g} \mathrm{PI}$ in $50 \mu \mathrm{l}$ sterile phosphate buffered saline (PBS)). A total of 33 NOD female mice were used in this study; 13 were treated with $\mathrm{PI}$-coated MNs, 9 were treated with a conventional ID injection of PI and 11 were left untreated (controls).

2.7.2 Preparation of Carboxyfluorescein Succinimidyl Ester (CFSE)-labelled G9 CD8 ${ }^{+} \mathrm{T}$ cells

G9Ca-/- NOD transgenic mouse spleens were harvested and processed into a single cell suspension using a tissue homogenizer (a total of 53 mouse spleens were used in these studies). Following osmotic lysis of the erythrocytes, the splenocyte suspension was filtered through a $40 \mu \mathrm{m}$ cell strainer and centrifuged. CD8 ${ }^{+} \mathrm{T}$ cells were isolated by negative selection using the MACS CD8a ${ }^{+} \mathrm{T}$ cell isolation kit, according to the manufacturer's instructions. Using the Vybrant ${ }^{\text {TM }}$ Carboxyfluorescein Diacetate Succinimidyl Ester (CFDA SE) Cell Tracer Kit (Thermo Fisher Scientific, UK) the isolated $\mathrm{G} 9 \mathrm{CD}^{+} \mathrm{T}$ cells were incubated with $2 \mu \mathrm{M}$ CFDA SE with the final cell suspension being produced in sterile saline (sodium chloride $0.9 \%$ ) to a concentration of $4 \times 10^{7} / \mathrm{ml}$. CFDA SE is converted intracellularly into fluorescent CFSE and the CFSE-labelled G9 $\mathrm{CD}^{+} \mathrm{T}$ cells can be detected by flow cytometry.

2.7.3 Detection of CFSE-labelled G9 $\mathrm{CD}^{+} \mathrm{T}$ cells in the LNs and spleen of Pl-treated mice

Twenty-four hours after coated MN treatment, NOD mice received an IV cell transfer of $8 \times 10^{6}$ CFSE-labelled G9 CD8 ${ }^{+}$T cells in an injection volume of $200 \mu$, into the tail vein using a 27G hypodermic needle. NOD mice were then sacrificed 96 hours post- 
cell transfer and the spleens were harvested and processed to a splenocyte suspension (section 2.7.2). The axillary LNs (AxLNs) were also harvested and mechanically dissociated to release the cells, which were subsequently filtered through a $40 \mu \mathrm{m}$ cell strainer. Cell samples were analyzed on a BD LSRFortessa ${ }^{\mathrm{TM}}$ (BD Biosciences, UK) and data was analyzed using FlowJo version X.0.7 (Tree Star, Inc., USA). Dilution of the CFSE signal in flow cytometric analysis is an indicator of cell proliferation.

\subsection{Statistical analysis}

Statistical analyses for all studies were conducted using GraphPad Prism 6 (GraphPad Software Inc., USA) and/ or Microsoft Excel (Microsoft Corporation, USA). One-way ANOVA with appropriate post-hoc tests were conducted to determine statistical significance. Data are expressed as mean \pm standard deviation.

\section{Results}

\subsection{Quantification of the dose of PI coated on MNs}

Three rows of MNs, forming an array of 30 needles, were manually coated with $0.5 \mu \mathrm{l}$ of a coating formulation, containing $44.6 \pm 4.3 \mu \mathrm{g}$ of PI. Recovery of the coated dose indicated that $31.5 \pm 2.9 \mu \mathrm{g}$ of $\mathrm{PI}$ was coated on MNs, a coating efficiency of $70.6 \pm$ $6.5 \%$. A mass of $5.2 \pm 3.3 \mu \mathrm{g}$ was recovered from the coating apparatus (Fig. 2).

\subsection{Puncture performance of PI-coated MNs in skin}

Arrays of $30 \mathrm{PI}$-coated MNs were applied to the pinched scruff of murine skin. In all cases, this resulted in two sets of puncture marks; the entry points of individual MNs at the application site and exit points on the adjacent side of the skin fold (Fig. 3). Upon application, MN projections therefore span all layers of the folded skin. The frequency of puncture at the $\mathrm{MN}$ application site ranged from 26 to 30 out of 30 potential punctures $(95.6 \pm 5.8 \%$ puncture; $n=6)$ and in the adjacent skin fold from 19 to 30 out of 30 ( $86.1 \pm 17.8 \%$ puncture; $n=6)$. MN puncture at the application site was therefore more reproducible than in the adjacent skin.

\subsection{Intradermal deposition of PI from $\mathrm{PI}$-coated $\mathrm{MNs}$ in vivo}

Arrays of Pl-coated MNs were applied to the shaved skin at the scruff of the neck of live, anaesthetized mice and held in place for 1, 2.5 or 10 minutes. Control MNs were coated but not applied to skin and the coated material was quantified in parallel. The 
delivery efficiency after 1 minute of $\mathrm{MN}$ application was $77.5 \pm 17.3 \%$. This increased to $86.1 \pm 4.4 \%$ upon a 2.5 minute application and was $75.2 \pm 24.7 \%$ following 10 minutes of application. There was no significant difference in the mean dose delivered following different durations of application ( $p>0.05$ ), however 2.5 minutes was the most reproducible $(28.9 \pm 1.5 \mu \mathrm{g})$ of the times that were tested (Fig. 4). Whilst this may not be statistically significant, it was used to guide the duration of application in subsequent MN application studies.

SEM images of coated MNs both before (Fig. 5A, B) and after (Fig. 5C, D) a 2.5 minute application to murine skin also confirmed that the majority of the PI coating was removed from the MN surface following treatment. Small darkened areas were visible on the MN surface following application, but these are present on both coated and non-coated MNs and are indicative of the deposition of biological material from the murine skin (Fig. 5G, H).

3.4 Trafficking of MN-administered PI to the draining (axillary) LNs and stimulation of T cells in vivo

G9 CD8 ${ }^{+}$T cells recognize the B15-23 peptide of insulin (and PI) (Wong et al. 2009) when presented by DCs and they proliferate in response (supplementary figure 2). Thus, the G9 CD8 ${ }^{+} \mathrm{T}$ cells were used in vivo to detect if $\mathrm{PI}$, locally delivered to the skin using MNs or a conventional intradermal (ID) injection, was transferred to the draining (axillary) lymph nodes (AxLNs). Proliferation of adoptively transferred G9 $\mathrm{CD}^{+} \mathrm{T}$ cells was clearly detectable in the AxLNs in the MN treated group but not in the ID-treated and untreated groups (Fig. 6B). Results from multiple in vivo experiments (Fig 6C) show that conventional ID injection of PI did not induce significant proliferation of transferred $\mathrm{G} 9 \mathrm{CD} 8^{+} \mathrm{T}$ cells in the AxLNs, thus indicating that the locally administered PI antigen did not drain to the local lymph nodes, either with or without the aid of APCs. However, MN-delivered PI stimulated significantly greater proliferation in the AxLNs than both the untreated control $(p \leq 0.0001)$ and the conventional ID PI injection ( $p \leq 0.001$ ) (Fig. $6 \mathrm{C}$ ). A very low level of proliferation of the transferred G9 CD8 ${ }^{+} \mathrm{T}$ cells with no difference between the groups was observed in the spleen (data not shown) $(p>0.05)$.

\section{Discussion}

The multi-epitope PI protein has potential clinical advantages over existing candidates for ASI of T1D in terms of both efficacy and suitability in a patient population with heterogenous HLA status. The skin is a particularly attractive target 
organ for immunotherapies but delivery of a tolerogenic drug candidate into the tissue necessitates use of a delivery system that minimizes the local inflammatory response. This study has therefore explored the potential of coated MNs as an appropriate delivery system for PI.

The initial suitability of a drug delivery system for an active pharmaceutical ingredient (API) is determined, in part, by the dosing requirements for the API and the capacity of the dosage form. The micron scale features of a coated MN device have typically confined their clinical utility to microgram doses of drugs and vaccines (Gill and Prausnitz 2007) although there are efforts to increase the capacity of the device (Donnelly et el. 2012). The optimal intradermal dose of PI for ASI is unknown; however, $10 \mu \mathrm{g}$ has been conventionally used in single peptide ASI studies for treatment of T1D (Gibson et al. 2015; Zhao 2016; Alhadj Ali et al. 2017). The molecular weight of the $\mathrm{PI}$ protein is typically 6-13 times greater than the relevant single peptide autoantigens but this is unlikely to translate to a directly proportional increase in dose, because the multi-epitope protein is predicted to provide improvements in both efficacy and potency. Targeted delivery of the therapeutic is also predicted to reduce the dosing requirement and therefore, based on these assumptions, $30 \mu \mathrm{g}$ of $\mathrm{API}$ on a $30 \mathrm{MN}$ array was selected as a reasonable dose for this proof-of-concept study to demonstrate the suitability and functionality of the coated MN system for PI delivery into skin. Future pre-clinical studies will need to optimize this dose and this may include a flexible dosing strategy, which has been used in related ASI studies (Burton et al. 2014) to treat an experimental autoimmune encephalomyelitis (EAE) mouse model of multiple sclerosis with an escalating dose immunotherapy (a 10-fold increase in the dose of autoantigen every 3-4 days from 0.08 to $80 \mu \mathrm{g}$ ) to achieve both a reduction in the proliferative capacity of $\mathrm{CD}^{+} \mathrm{T}$ cells and induction of $\mathrm{CD}^{+}{ }^{+} \mathrm{T}$ cells with a regulatory phenotype. The coated $\mathrm{MN}$ system lends itself to this potential requirement for flexibility, with an escalated or tapered dosing regimen being accommodated by simply changing the number of needles on a coated $\mathrm{MN}$ device and/or modifying their loading.

The coating process for MNs can be technically challenging and high levels of reproducibility in the final dosage form will be key to clinical efficacy, safety and regulatory acceptance. Coating formulations for MNs therefore often include a blend of surfactants, viscosity enhancers and stabilizers, in addition to the API and solvent (Gill and Prausnitz 2007; Kim et al. 2010; Ameri et al. 2014), to facilitate rapid and uniform coating. However, the micron-scale of $\mathrm{MNs}$ and the importance of 
maintaining a sharp tip (to enable skin puncture and reduce deposition of the coated formulation on the skin surface (Gill and Prausnitz 2007)) restricts the volume of the coating formulation that can be applied to each needle. The volume and quantity of excipients in a MN coating formulation must therefore be minimized to ensure there is sufficient capacity for the API, and in this case the dose that is required $(1 \mu \mathrm{g} /$ microneedle). Polysorbate 80 is a low toxicity excipient (Agarkhed et al. 2013) that has been used for its surfactant properties in MN spray-coating formulations (McGrath et al. 2011; Vrdoljak et al. 2012), as a stabilizer in protein formulations (Agarkhed et al. 2013), as a solubilising excipient in oral dosage forms and in injectable pharmaceutical formulations (Strickley 2004). Inclusion of this non-ionic surfactant in the coating formulation resulted in a uniform coating that was restricted to the MN shaft (Fig. 5) and facilitated reproducible PI dosing (Fig. 4). The low concentration of this excipient $(0.5 \%)$ in the $\mathrm{PI}$ coating formulation, which is comparable to that used in previous studies (0.5-1\%) (McGrath et al. 2011; Vrdoljak et al. 2012), maximized capacity for the high molecular weight API and minimized MN loading. PI coated MNs therefore retained the sharp tip of their uncoated counterparts (Fig. 5) and penetration into the murine model was not impeded (Fig. 3).

Acetic acid (AA) was identified as an appropriate solvent for PI (Linde et al. 1991; Landreh et al. 2012) and has been previously used in the MN coating formulations of pancreatic autoantigens (Zhao et al. 2016). However, in an acidic environment, acidic amino acids become more protonated, disrupting salt bridges and altering the secondary structure of a protein (Leon et al. 2019). The resultant unfolding of the protein exposes hydrophobic residues to the external environment (Choi et al. 2014). It is established that acidic $\mathrm{pH}$ can denature insulin. Insulin exists in unstable dimeric and monomeric forms at low pH (Nielsen et al. 2001) which are likely to aggregate and lead to fibrillation (Selivanova and Galzitskaya 2012; lannuzzi et al. 2017), as a result of the exposure of hydrophobic residues of the protein (Nielsen et al. 2001). Hydrophobicity may mask the protein from the immune system, an effect demonstrated by fungal spores covered in hydrophobin (Aimanianda et al. 2009), which is an unwanted effect in ASI. However, for the purposes of peripheral tolerance induction, retention of tertiary or secondary structure of $\mathrm{PI}$ is not crucial during the formulation, coating and delivery process because APCs will ultimately process the peptides. In fact, the unfolding of $\mathrm{PI}$ during denaturation may be beneficial as it would make the cognate epitopes more readily available to the APCs. It is evident from the immunological data that, in this study, the acidity of the 
formulation was not detrimental and the primary structure and autoantigenic epitopes of PI were retained throughout the coating and delivery process."

At this early stage of development, a manual coating method was selected as a simple and rapid method that could be used to produce a uniform and reproducible coating using FDA approved excipients. However, only $70 \%$ of $\mathrm{PI}$ in the coating solution was deposited on the MN device. Therefore, whilst reproducibility is the key parameter for patient safety and efficacy, translation to the commercial scale would require a greater understanding of the process parameters and subsequent reductions to the $30 \%$ waste. An increase in coating efficiency can be achieved through automation of the coating process to provide a more rapid, high-throughput method of accurate and reproducible coating (Gill and Prausnitz 2007) with reduced wastage and the potential for scale-up. A MN coating process, in which MNs are coated using a roller drum has been used to successfully coat proteins with a higher molecular weight than proinsulin (Peters et al. 2012; Ameri et al 2014) and a coating method akin to inkjet printing has enabled MNs to be coated with insulin in a finely controlled, highly accurate and reproducible automated process with minimal wastage (Ross et al. 2015; Pere et al. 2018). Automated dip-coating devices have also successfully coated individual MNs in an array with different drug formulations, thus allowing the co-delivery of multiple agents with different characteristics ( $\mathrm{Li}$ et al 2018). This innovative method may be useful in future studies if co-administration of a tolerogenic adjuvant were required with a PI-coated $\mathrm{MN}$ treatment. These examples exemplify the potential to scale-up and also improve the efficiencies of a dip-coating process for a MN product.

One advantage of coated MN systems compared to liquid formulations is enhanced stability of the dried form of the active pharmaceutical ingredient (Kapoor et al. 2019). The stability of a number of MN-coated therapeutics, including insulin (Pere et al. 2018), have been studied and published data indicates stability times from weeks to months (Peters et al. 2012; Kim et al. 2015; Baek et al. 2017; Kapoor et al. 2019). In this study, PI coating formulations were freshly prepared and MNs were coated within hours of their use but future studies should evaluate the physical and chemical stability of the system in controlled conditions for extended storage times.

Mouse skin is significantly thinner ( $>10 x)$ than its human counterpart (Wei et al. 2017) and the depth of the cellular region of the tissue, i.e. the viable epidermis, is just $10 \mu \mathrm{m}$ in the mouse, compared to $100 \mu \mathrm{m}$ in the human (Hansen et al. 1984; 
Prausnitz and Langer 2008). To account, in part, for these architectural differences the PI-coated MNs were applied to folded skin on the scruff of the neck of the mouse. This served two purposes. The first was to limit the extent of MN protrusion, i.e. the MNs were retained predominantly in skin tissue and were prevented from extending into the underlying muscle, and the second was to increase the numbers of APCs in the skin that are exposed to PI. Skin puncture studies using the PI-coated MNs indicated this strategy was successful and therefore deposition of the cargo in the double thickness epidermis-dermis may have contributed to enhanced uptake of PI by the local immune cells, compared to ID injection.

The duration of application had no significant effect on the mass of PI delivered into mouse skin, within the range of times that were tested (1-10 minutes). Deposition and/or dissolution of the $\mathrm{PI}$ formulation from the surface of the stainless steel $\mathrm{MN}$ is therefore relatively rapid. In this study a 2.5 minute application was used because, while it may not be perceived as the optimum application time for the patient, i.e. the minimum time, it provided confidence that the entire MN cargo was deposited in skin following removal of the device from the tissue. A range of application times $(<3$ minutes to 1 hour) have been reported for MN coated APIs in murine, guinea pig and rat skin (Cormier et al. 2004; Kim et al. 2010; Chong et al. 2013; Ameri et al. 2014; Moreno et al. 2017; Shakya et al. 2017) and delivery efficiencies vary widely (37$>90 \%$ ). The duration of application required for MN delivery systems is determined, in part, by the solubility of the API and the coating formulation (Zhao et al. 2016; Zhao et al. 2017), and so poorly soluble macromolecular therapeutics, such as plasmid DNA, may require extended application times (Moreno et al. 2017). However, there are many factors that determine application times, including the skin model that is used, the dimensions and design of the MNs and the method of application; application times for coated MN products are therefore likely to be system dependent. This study demonstrates that a PI-coated MN can deposit its cargo in murine skin with a reasonable delivery efficiency $(70 \%)$ and within a time frame that is predicted to be acceptable to a patient (1 minute).

Localized deposition of a reproducible and clinically relevant $\mathrm{PI}$ dose was a fundamental first step for a delivery system that aimed to target the immunocompetent resident skin cells; however it was the in vivo experiments using adoptively transferred CFSE-labelled $\mathrm{G} 9 \mathrm{CD}^{+} \mathrm{T}$ cells that provided evidence of cellular targeting and potential therapeutic utility (Fig. 6). The G9 CD8 ${ }^{+} \mathrm{T}$ cells used in these studies recognize a specific antigen fragment of PI (the insulin B15-23 
peptide) presented by APCs and proliferate in response to this. Therefore, the proliferation of these transferred, antigen-specific $\mathrm{T}$ cells, compared to the control, following treatment of the mice with Pl-coated MNs (Fig. 6) suggested successful delivery of the PI to APCs in the skin (LCs and/or dDCs), which had subsequently processed the PI, migrated to the AxLNs and presented it to the transferred, antigenspecific $\mathrm{T}$ cells. Most notably, this proliferation profile was not replicated in mice treated with an ID injection of $\mathrm{PI}$, thus supporting the hypothesis that the PI-coated MN system provided a more targeted delivery platform. Proliferation of the transferred, antigen-specific T cells in the spleen of PI-treated mice (both MN and ID) was comparable to the control (data not shown), providing evidence of localized rather than systemic delivery.

Previous studies delivering comparable therapeutic cargos via coated MNs have reported enhanced localization of material at the administration site and a prolonged skin residence time compared to conventional ID injections (Torrisi et al. 2013; Zhao et al. 2016; Zhao et al. 2017). Enhanced T cell proliferation in the AxLNs following $\mathrm{MN}$-mediated delivery of PI may be attributable to more localized deposition of the therapeutic cargo and/or delivery of the protein in a concentrated solid form rather than a bolus solution, since solid antigen is targeted and internalized by phagocytosis more efficiently than its soluble form (Snapper 2018). Delivery of a high molecular weight poorly soluble protein, such as $\mathrm{PI}$, into a confined region of the skin from a solid coating is likely to increase both the concentration and residency time within the local environment, resulting in an increase in the extent and duration of APC exposure to the autoantigen (Zhao et al. 2016) and the consequential stimulation and proliferation of $T$ cells in the AxLNs. Whilst the results confirm targeting of the local immune system and are indicative of the aforementioned delivery mechanism, an alternative hypothesis is that locally delivered PI was not captured by APCs but, instead, diffused directly into lymphatic vessels that drained to the AxLNs. Future studies could probe these mechanisms using a mouse model (CCR7-deficient mouse) which lacks the $\mathrm{C}-\mathrm{C}$ chemokine receptor (CCR7) protein that is necessary for DC migration from the skin to the draining LNs (Ohl et al. 2004).

Whilst this in vivo data provides substantive evidence to support future development of $\mathrm{PI}$-coated MNs, the local immunological response to the PI-coated MN intervention was associated with variability (the percentage of $T$ cell proliferation ranging from $4.6 \%$ to $29.1 \%$ (Fig. $6 \mathrm{C}$ )). The source of this variability may be related to the drug delivery system, i.e. the manual coating procedure and manual application of the 
MNs, and/or the inherent variability within an in vivo model. Further studies are therefore required to optimize elements of the drug delivery system, e.g. dosing levels, intervals, frequency and duration, and to evaluate how the local immune response to $\mathrm{PI}$-coated $\mathrm{MN}$ treatment translates to clinical efficacy, i.e. the induction of tolerance in vivo.

\section{Conclusion}

This study demonstrates, for the first time, that PI can be uniformly and reproducibly coated on solid MNs to produce a delivery system that delivers a therapeutically relevant dose of $\mathrm{PI}$ into the skin to stimulate a local immune response. The $\mathrm{PI}$-coated MN delivery system has discernible targeting advantages compared to a conventional ID injection and has demonstrated significant therapeutic potential in ASI for T1D; the full protein PI antigen provides a multi-epitope antigen that may facilitate clinical applicability across a wide population and the delivery system minimizes inflammation in the local environment, thus maximising the potential to induce tolerance. Future studies should evaluate the potential of the PI-coated MN system as a means to induce tolerance for T1D.

Information on the data underpinning the results presented here, including how to access them, can be found in the Cardiff University data catalogue at http://doi.org/10.17035/d.2019.0077300268.

\section{Acknowledgements}

This work is part of the EE-ASI European research network (Collaborative Project) supported by the European Commission under the Health Cooperation Work Programme of the 7th Framework Programme (Grant agreement $n^{\circ}$ 305305). The work was also supported by the Engineering and Physical Sciences Research Council [grant number EP/M50631X/1] and the Juvenile Diabetes Research Foundation [grant number 17-2014-2].

\section{References}

Agarkhed, M. et al. 2013. Effect of polysorbate 80 concentration on thermal and photostability of a monoclonal antibody. AAPS PharmSciTech 14(1), pp. 1-9. doi: 10.1208/s12249-012-9878-0. 
Aimanianda, V. et al. 2009. Surface hydrophobin prevents immune recognition of airborne fungal spores. Nature 460 (7259), pp. 1117-1121. doi: $10.1038 /$ nature08264.

Akbarpour, M. et al. 2015. Insulin B chain 9-23 gene transfer to hepatocytes protects from type 1 diabetes by inducing Ag-specific FoxP3+ Tregs. Sci Transl Med 7(289), 289ra281. doi: 10.1126/scitransImed.aaa3032.

Alhadj Ali, M. et al. 2017. Metabolic and immune effects of immunotherapy with proinsulin peptide in human new-onset type 1 diabetes. Sci Transl Med 9(402), eaaf7779. doi: 10.1126/scitranslmed.aaf7779.

Ali, M.A. et al. 2015. Topical steroid therapy induces pro-tolerogenic changes in Langerhans cells in human skin. Immunology 146(3), pp. 411-422. doi: 10.1111/imm.12518.

Ameri, M. et al. 2014. Human Growth Hormone Delivery with a Microneedle Transdermal System: Preclinical Formulation, Stability, Delivery and PK of Therapeutically Relevant Doses. Pharmaceutics 6(2), pp. 220-234. doi: 10.3390/pharmaceutics6020220.

Arai, K. et al. 2018. Characterization and optimization of two-chain folding pathways of insulin via native chain assembly. Commun Chem 1, 26. doi:10.1038/s42004-0180024-0.

Arce-Sillas, A. et al. 2016. Regulatory T Cells: Molecular Actions on Effector Cells in Immune Regulation. J Immunol Res 2016, 1720827. doi: 10.1155/2016/1720827.

Arya, J. et al. 2017. Tolerability, usability and acceptability of dissolving microneedle patch administration in human subjects. Biomaterials 128, pp. 1-7. doi: 10.1016/j.biomaterials.2017.02.040.

Baek, S.H. et al. 2017. Drug-coated microneedles for rapid and painless local anesthesia. Biomed Microdevices 19 (1), 2. doi: 10.1007/s10544-016-0144-1. 
Burrack, A.L. et al. 2017. T Cell-Mediated Beta Cell Destruction: Autoimmunity and Alloimmunity in the Context of Type 1 Diabetes. Front Endocrinol (Lausanne) 8, 343. doi: 10.3389/fendo.2017.00343.

Burton, B.R. et al. 2014. Sequential transcriptional changes dictate safe and effective antigen-specific immunotherapy. Nat Commun 5, 4741. doi: 10.1038/ncomms5741.

Chen, C. et al. 2017. Human beta cell mass and function in diabetes: Recent advances in knowledge and technologies to understand disease pathogenesis. Mol Metab 6(9), pp. 943-957. doi: 10.1016/j.molmet.2017.06.019.

Chen, W. et al. 2001. Evidence that a peptide spanning the B-C junction of proinsulin is an early autoantigen epitope in the pathogenesis of type 1 diabetes. $J$ Immunol 167(9), pp. 4926-4935. doi: 10.4049/jimmunol.167.9.4926.

Choi, T.S. et al. 2014. Amyloid fibrillation of insulin under water-limited conditions. Biophys J 107 (8), pp. 1939-1949. doi: 10.1016/j.bpj.2014.09.008.

Chong, R. H. E. et al. 2013. Gene silencing following siRNA delivery to skin via coated steel microneedles: In vitro and in vivo proof-of-concept. $J$ Control Release 166(3), pp. 211-219. doi: 10.1016/j.jconrel.2012.12.030.

Cormier, M. et al. 2004. Transdermal delivery of desmopressin using a coated microneedle array patch system. J Control Release 97(3), pp. 503-511. doi: 10.1016/j.jconrel.2004.04.003.

Dalod, M. et al. 2014. Dendritic cell maturation: functional specialization through signaling specificity and transcriptional programming. EMBO J 33(10), pp. 11041116. doi: 10.1002/embj.201488027.

De Groot, A.S. et al. 2019. Therapeutic administration of Tregitope-Human Albumin Fusion with Insulin Peptides to promote Antigen-Specific Adaptive Tolerance Induction. Sci Rep 9 (1), 16103. doi:10.1038/s41598-019-52331-1.

Di Lorenzo, T. P. et al. 2007. Translational mini-review series on type 1 diabetes: Systematic analysis of $\mathrm{T}$ cell epitopes in autoimmune diabetes. Clin Exp Immunol 148(1), pp. 1-16. doi: 10.1111/j.1365-2249.2006.03244.x. 
Donnelly, R.F. et al 2012. Hydrogel-Forming Microneedle Arrays for Enhanced Transdermal Drug Delivery. Adv Funct Mater 22(23), pp. 4879-4890. doi: 10.1002/adfm.201200864.

Dul M. et al. 2017. Hydrodynamic gene delivery in human skin using a hollow microneedle device. $J$ Control Release 265, pp. 120-131. doi: 10.1016/j.jconrel.2017.02.028.

Dul, M. et al. 2019. Conjugation of a peptide autoantigen to gold nanoparticles for intradermally administered antigen specific immunotherapy. Int $J$ Pharm 562, pp. 303-312. doi: 10.1016/j.ijpharm.2019.03.041.

Gibson, V. B. et al. 2015. Proinsulin multi-peptide immunotherapy induces antigenspecific regulatory $\mathrm{T}$ cells and limits autoimmunity in a humanized model. Clin Exp Immunol 182(3), pp. 251-260. doi: 10.1111/cei.12687.

Gill, H. S. and Prausnitz, M. R. 2007. Coating formulations for microneedles. Pharm Res 24(7), pp. 1369-1380. doi: 10.1007/s11095-007-9286-4.

Gupta, J. et al. 2011. Rapid pharmacokinetics of intradermal insulin administered using microneedles in type 1 diabetes subjects. Diabetes Technol Therapy 13(4), pp. 451-456. doi: 10.1089/dia.2010.0204.

Hansen, L. S. et al. 1984. The influence of the hair cycle on the thickness of mouse skin. Anat Rec 210(4), pp. 569-573. doi: 10.1002/ar.1092100404.

Haq, M. I. et al. 2009. Clinical administration of microneedles: skin puncture, pain and sensation. Biomed Microdevices 11(1), pp. 35-47. doi: 10.1007/s10544-0089208-1.

Ho, A.W. and Kupper, T.S. 2019. T cells and the skin: from protective immunity to inflammatory skin disorders. Nat Rev Immunol 19 (8), pp. 490-502. doi:10.1038/s41577-019-0162-3. 
lannuzzi, C. et al. 2017. Insights into Insulin Fibril Assembly at Physiological and Acidic pH and Related Amyloid Intrinsic Fluorescence. Int. J. Mol. Sci. 18(12), 2551. doi: 10.3390/ijms18122551.

Iberg, C. A. et al. 2017. Dendritic Cells As Inducers of Peripheral Tolerance. Trends Immunol 38(11), pp. 793-804. doi: 10.1016/j.it.2017.07.007.

Jeong, H.R. et al. 2018. Local dermal delivery of cyclosporin A, a hydrophobic and high molecular weight drug, using dissolving microneedles. Eur J Pharm Biopharm 127, pp. 237-243. doi: 10.1016/j.ejpb.2018.02.014.

Kapoor, Y. et al. 2019. Coated microneedles for transdermal delivery of a potent pharmaceutical peptide. Biomed Microdevices 22(1), 7. doi: 10.1007/s10544-0190462-1.

Kent, S.C. et al. 2017. Deciphering the Pathogenesis of Human Type 1 Diabetes (T1D) by Interrogating T Cells from the "Scene of the Crime". Curr Diab Rep 17(10), 95. doi: 10.1007/s11892-017-0915-y.

Kim, Y. C. et al. 2010. Formulation and coating of microneedles with inactivated influenza virus to improve vaccine stability and immunogenicity. $J$ Control Release 142(2), pp. 187-195. doi: 10.1016/j.jconrel.2009.10.013.

Kim, M.C. et al. 2015. Microneedle patch delivery to the skin of virus-like particles containing heterologous M2e extracellular domains of influenza virus induces broad heterosubtypic cross-protection. J Control Release 210, pp. 208-216. doi: 10.1016/j.jconrel.2015.05.278.

Kroger, C. J. et al. 2018. Therapies to Suppress $\beta$ Cell Autoimmunity in Type 1 Diabetes. Front Immunol 9, 1891. doi: 10.3389/fimmu.2018.01891.

Landreh, M. et al. 2012. Insulin solubility transitions by $\mathrm{pH}$-dependent interactions with proinsulin C-peptide. FEBS J 279(24), pp. 4589-4597. doi: 10.1111/febs.12045.

Lau, S. et al. 2017. Multilayered pyramidal dissolving microneedle patches with flexible pedestals for improving effective drug delivery. J Control Release 265, pp. 113-119. doi: 10.1016/j.jconrel.2016.08.031. 
Leon, M.A. et al. 2019. Soluble Antigen Arrays for Selective Desensitization of Insulin-Reactive B Cells. Mol Pharm 16(4), pp. 1563-1572. doi: 10.1021/acs.molpharmaceut.8b01250.

Leone, M. et al. 2017. Dissolving Microneedle Patches for Dermal Vaccination. Pharm Res 34(11), pp. 2223-2240. doi: 10.1007/s11095-017-2223-2.

$\mathrm{Li}$, S. et al. 2018. Individually coated microneedles for co-delivery of multiple compounds with different properties. Drug Deliv Transl Res 8(5), pp. 1043-1052. doi: 10.1007/s13346-018-0549-x.

Linde, S. et al. 1991. Separation and quantitation of serum proinsulin and proinsulin intermediates in humans. J Chromatogr 548(1-2), pp. 371-380. doi: 10.1016/s00219673(01)88620-9.

Liu, L. et al. 2003. Toll-like receptor-9 induced by physical trauma mediates release of cytokines following exposure to CpG motif in mouse skin. Immunology 110(3), pp. 341-347. doi: 10.1046/j.1365-2567.2003.01739.x.

Liu, et al. 2005. CpG motif acts as a 'danger signal' and provides a T helper type 1biased microenvironment for DNA vaccination. Immunology 115(2), pp. 223-230. doi: 10.1111/j.1365-2567.2005.02150.x.

McGrath, M. G. et al. 2011. Determination of parameters for successful spray coating of silicon microneedle arrays. Int $J$ Pharm 415(1-2), pp. 140-149. doi: 10.1016/j.jpharm.2011.05.064.

Michels, A.W. et al. 2017. Islet-Derived CD4 T Cells Targeting Proinsulin in Human Autoimmune Diabetes. Diabetes 66(3), pp. 722-734. doi: 10.2337/db16-1025.

Moreno, E. et al. 2017. Skin vaccination using microneedles coated with a plasmid DNA cocktail encoding nucleosomal histones of Leishmania spp. Int J Pharm 533(1), pp. 236-244. doi: 10.1016/j.ijpharm.2017.09.055. 
Naito, C. et al. 2018. Self-Dissolving Microneedle Arrays for Transdermal Absorption Enhancement of Human Parathyroid Hormone (1-34). Pharmaceutics 10(4), 215. doi: 10.3390/pharmaceutics 10040215 .

Nielsen, L. et al. 2001. Effect of environmental factors on the kinetics of insulin fibril formation: elucidation of the molecular mechanism. Biochemistry 40(20), pp. 60366046. doi: 10.1021/bi002555c.

Ohl, L. et al. 2004. CCR7 governs skin dendritic cell migration under inflammatory and steady-state conditions. Immunity 21(2), pp. 279-288. doi: 10.1016/j.immuni.2004.06.014.

Palylyk-Colwell, E. and Ford, C. 2017. A Transdermal Glucagon Patch for Severe Hypoglycemia. In: CADTH Issues in Emerging Health Technologies. Ottawa (ON): Canadian Agency for Drugs and Technologies in Health. Available at: https://www.ncbi.nlm.nih.gov/books/NBK481476/\#transdermal_glucagon_hypoglyce mia.rl [Accessed: 3 Jan 2020].

Pathiraja, V. et al. 2015. Proinsulin-specific, HLA-DQ8, and HLA-DQ8-transdimerrestricted CD4+ T cells infiltrate islets in type 1 diabetes. Diabetes 64(1), pp. 172182. doi: $10.2337 / \mathrm{db} 14-0858$.

Pere, C.P.P. et al. 2018. 3D printed microneedles for insulin skin delivery. Int $J$ Pharm 544(2), pp. 425-432. doi: 10.1016/j.ijpharm.2018.03.031.

Peters, E. E. et al. 2012. Erythropoietin-coated ZP-microneedle transdermal system: preclinical formulation, stability, and delivery. Pharm Res 29(6), pp. 1618-1626. doi: 10.1007/s11095-012-0674-z.

Petzold, C. et al. 2010. Dendritic cell-targeted pancreatic beta-cell antigen leads to conversion of self-reactive CD4(+) T cells into regulatory $\mathrm{T}$ cells and promotes immunotolerance in NOD mice. Rev Diabet Stud 7(1), pp. 47-61. doi: 10.1900/rds.2010.7.47.

Prausnitz, M. R. and Langer, R. 2008. Transdermal drug delivery. Nat Biotechnol 26(11), pp. 1261-1268. doi: 10.1038/nbt.1504. 
Prausnitz, M.R. 2017. Engineering microneedle patches for vaccination and drug delivery to skin. Annu Rev Chem Biomol Eng 8, pp. 177-200. doi: 10.1146/annurevchembioeng-060816-101514.

Ross, S. et al. 2015. Inkjet printing of insulin microneedles for transdermal delivery. Drug Deliv Transl Res 5(4), pp. 451-461. doi: 10.1007/s13346-015-0251-1.

Selinova, O.M. and Galzitskaya, O.V. 2012. Structural polymorphism and possible pathways of amyloid fibril formation on the example of insulin protein. Biochemistry (Mosc) 77(11), pp. 1237-1247. doi: 10.1134/S0006297912110028.

Shakya, A. K. et al. 2017. Cutaneous vaccination with coated microneedles prevents development of airway allergy. $J$ Control Release 265, pp. 75-82. doi: 10.1016/j.jconrel.2017.08.012.

Shklovskaya, E. et al. 2011. Langerhans cells are precommitted to immune tolerance induction. Proc Natl Acad Sci U S A 108(44), pp. 18049-18054. doi: 10.1073/pnas.1110076108.

Smith, E. L. and Peakman, M. 2018. Peptide immunotherapy for type 1 diabetesclinical advances. Front Immunol 9, 392. doi: 10.3389/fimmu.2018.00392.

Snapper, C.M. 2018. Distinct immunologic properties of soluble versus particulate antigens. Front Immunol 9, 598. doi : 10.3389/fimmu.2018.00598.

So, M. et al. 2018. Proinsulin C-peptide is an autoantigen in people with type 1 diabetes. Proc Natl Acad Sci U S A 115(42), pp. 10732-10737. doi: 10.1073/pnas.1809208115.

Steck, A.K. and Rewers, M.J. 2011. Genetics of Type 1 Diabetes. Clin Chem 57(2), pp. 176-185. doi : 10.1373/clinchem.2010.148221.

Steinman, R. M. et al. 2003. Dendritic cell function in vivo during the steady state: a role in peripheral tolerance. Ann N Y Acad Sci 987(1), pp. 15-25. doi: 10.1111/j.17496632.2003.tb06029.x. 
Strickley, R. G. 2004. Solubilizing excipients in oral and injectable formulations. Pharm Res 21(2), pp. 201-230. doi: 10.1023/b:pham.0000016235.32639.23.

Tian, J. et al. 2014. Combined therapy with GABA and proinsulin/alum acts synergistically to restore long-term normoglycemia by modulating T-cell autoimmunity and promoting beta-cell replication in newly diabetic NOD mice. Diabetes 63(9), pp. 3128-3134. doi: 10.2337/db13-1385.

Torrisi, B. M. et al. 2013. Pocketed microneedles for rapid delivery of a liquid-state botulinum toxin A formulation into human skin. J Control Release 165(2), pp. 146152. doi: 10.1016/j.jconrel.2012.11.010.

Turvey, M.E. et al. 2019. Microneedle-based intradermal delivery of stabilized dengue virus. Bioeng Transl Med 4(2), e10127. doi: 10.1002/btm2.10127.

Vrdoljak, A. et al. 2012. Coated microneedle arrays for transcutaneous delivery of live virus vaccines. $J$ Control Release 159(1), pp. 34-42. doi: 10.1016/j.jconrel.2011.12.026.

Wegmann, D.R. et al. 1994. Insulin-specific T cells are a predominant component of islet infiltrates in pre-diabetic NOD mice. Eur $J$ Immunol 24(8), pp. 1853-7. doi: 10.1002/eji.1830240820.

Wei, J.C.J. et al 2017. Allometric scaling of skin thickness, elasticity, viscoelasticity to mass for micro-medical device translation: from mice, rats, rabbits, pigs to humans. Sci Rep 7, 15885. doi: 10.1038/s41598-017-15830-7.

Wong, F. S. et al. 1996. CD8 T cell clones from young nonobese diabetic (NOD) islets can transfer rapid onset of diabetes in NOD mice in the absence of CD4 cells. $J$ Exp Med 183(1), pp. 67-76. doi: 10.1084/jem.183.1.67.

Wong, F. S. et al. 1999. Identification of an MHC class I-restricted autoantigen in type 1 diabetes by screening an organ-specific cDNA library. Nat Med 5(9), pp. 10261031. doi: $10.1038 / 12465$.

Wong, F. S. et al. 2009. Activation of insulin-reactive CD8 T-cells for development of autoimmune diabetes. Diabetes 58(5), pp. 1156-1164. doi: 10.2337/db08-0800. 
Yeste, A. et al. 2016. Tolerogenic nanoparticles inhibit T cell-mediated autoimmunity through SOCS2. Sci Signal 9(433), ra61. doi: 10.1126/scisignal.aad0612.

Yu, J. et al. 2015. Microneedle-array patches loaded with hypoxia-sensitive vesicles provide fast glucose-responsive insulin delivery. Proc Natl Acad Sci U S A 112(27), pp. 8260-8265. doi: 10.1073/pnas.1505405112.

Zhao, X. et al. 2016. Microneedle delivery of autoantigen for immunotherapy in type 1 diabetes. J Control Release 223, pp. 178-187. doi: 10.1016/j.jconrel.2015.12.040.

Zhao, X. et al. 2017. Formulation of hydrophobic peptides for skin delivery via coated microneedles. J Control Release 265, pp. 2-13. doi: 10.1016/j.jconrel.2017.03.015.

\section{Figure captions}

Fig. 1. Schematic representation of the method of MN application to murine skin. The shaved skin at the scruff of the neck was pinched, a supportive substrate was positioned behind the pinched skin and a Pl-coated array of $30 \mathrm{MNs}$ was applied.

Fig. 2. Quantification of MN coating with a PI formulation. Arrays of $30 \mathrm{MNs}$ were coated in $0.5 \mu$ l of a $P I$ coating formulation ( $P I$ in $0.2 \mathrm{MA} A$ ( $0.5 \%$ polysorbate 80 ), which was assayed by HPLC to determine the theoretical coated dose (control solution) ( $n=16)$. Coated MNs and pipette tips were washed to recover and quantify the PI (n=12). Individual data points are the mass of $P I$ in a single sample and horizontal lines are mean \pm standard deviation values.

Fig. 3. Puncture of murine skin by coated MNs. The MN-treated area of murine skin was stained with methylene blue, demonstrating the puncture insults that are related to both the entry of individual MNs and their exit on the adjacent skin surface.

Fig. 4. Quantification of PI delivery to murine skin from coated MNs in vivo.

The mass of PI coated on MNs was recovered and quantified by HPLC either 
immediately after coating (coated MNs control; $n=15$ ) or following application to the scruff of the neck of live, anaesthetized mice for 1, 2.5 or 10 minutes (undelivered PI remaining on MNs; $n=5)$. The dose of $\mathrm{Pl}$ delivered to skin is inferred from the expected coated dose and is, therefore, only an estimate of the "delivered dose". Individual data points are the mass of $P$ in a single sample and horizontal lines are mean \pm standard deviation values. There is no significant difference in the mass of $P I$ remaining on MNs following application for 1, 2.5 or 10 minutes ( $p>0.05$, One-way ANOVA with Tukey's multiple comparisons).

Fig. 5. SEM images to illustrate removal of PI following application to murine skin. (A-B) Pl-coated MNs prior to skin application. (C-D) Pl-coated MNs after application to murine skin for 2.5 minutes. (E-F) Non-coated MNs. (G-H) Non-coated $M N s$ after murine skin application for 2.5 minutes. Scale bars represent $500 \mu m(A, C$, $E, G)$ and $200 \mu m(B, D, F, H)$.

Fig. 6. Detecting G9 $\mathrm{CD}^{+} \mathrm{T}$ cell proliferation in the axillary lymph nodes of NOD mice in response to PI delivered by coated MNs or ID injection.

(A) NOD mice were injected with PI-coated MNs (MN), an ID injection of PI (ID) or had no treatment (control) and CFSE-labelled G9 CD8 ${ }^{+} T$ cells were adoptively transferred into the mice 24 hours later. After a further 96 hours, the percentage of CFSE $^{+}$G9 CD8 ${ }^{+} T$ cells that had proliferated in the AxLNs was quantified. (B) Illustrative examples of the flow cytometric plots used to detect proliferation of adoptively transferred G9 CD8 ${ }^{+} T$ cells in AxLNs. (C) Each data point represents G9 $C D 8^{+} T$ cell proliferation results from a single NOD mouse in the AxLNs and horizontal lines show the mean percentage of cell proliferation. Data combined from multiple experiments using a total of 33 NOD mice (Control $n=11$; $M N n=13$; ID $n=9$ ). Statistical analysis: ${ }^{* *} p \leq 0.001$ and ${ }^{* * *} p \leq 0.0001$, One-Way ANOVA with Tukey's multiple comparison. 


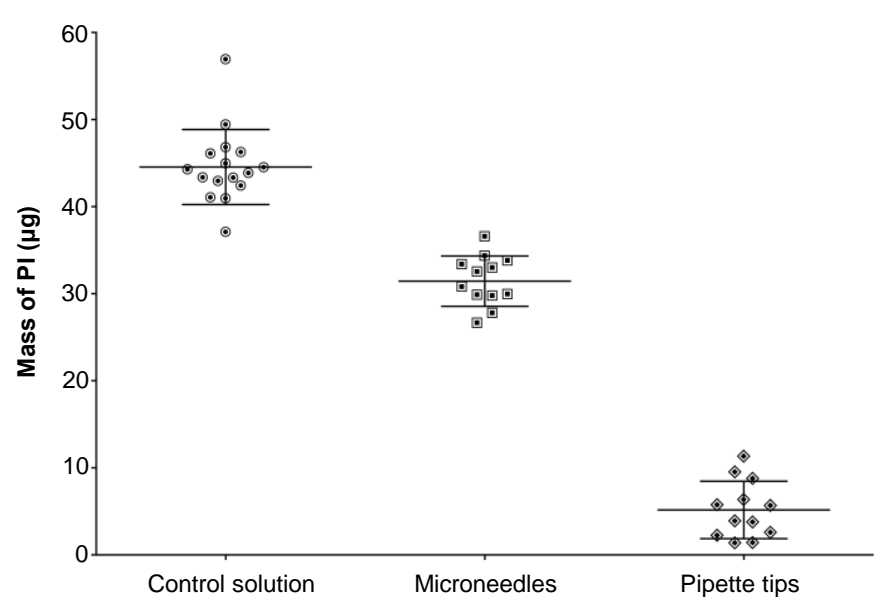

Fig.2.

Pipette tips 
Fig.4.

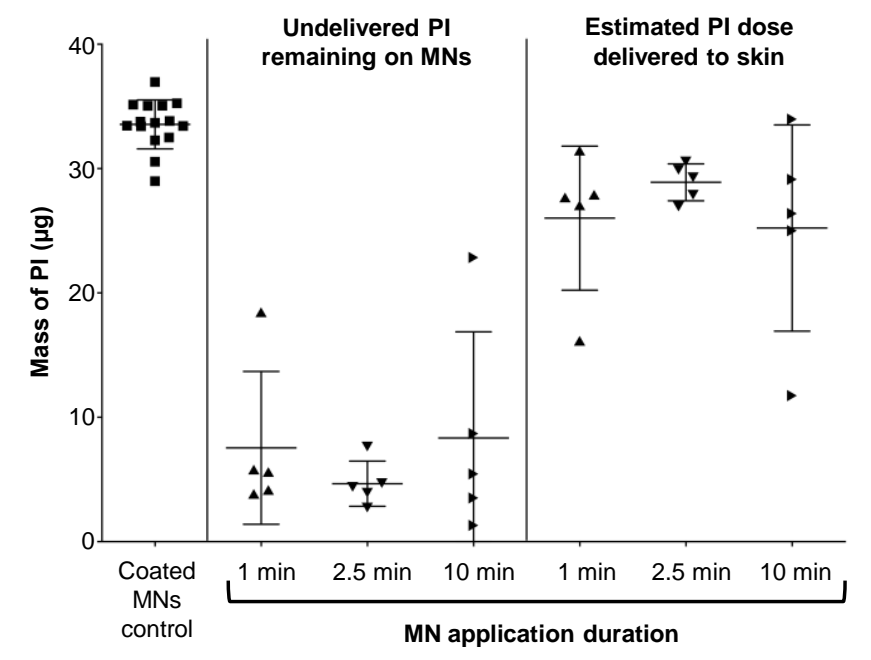




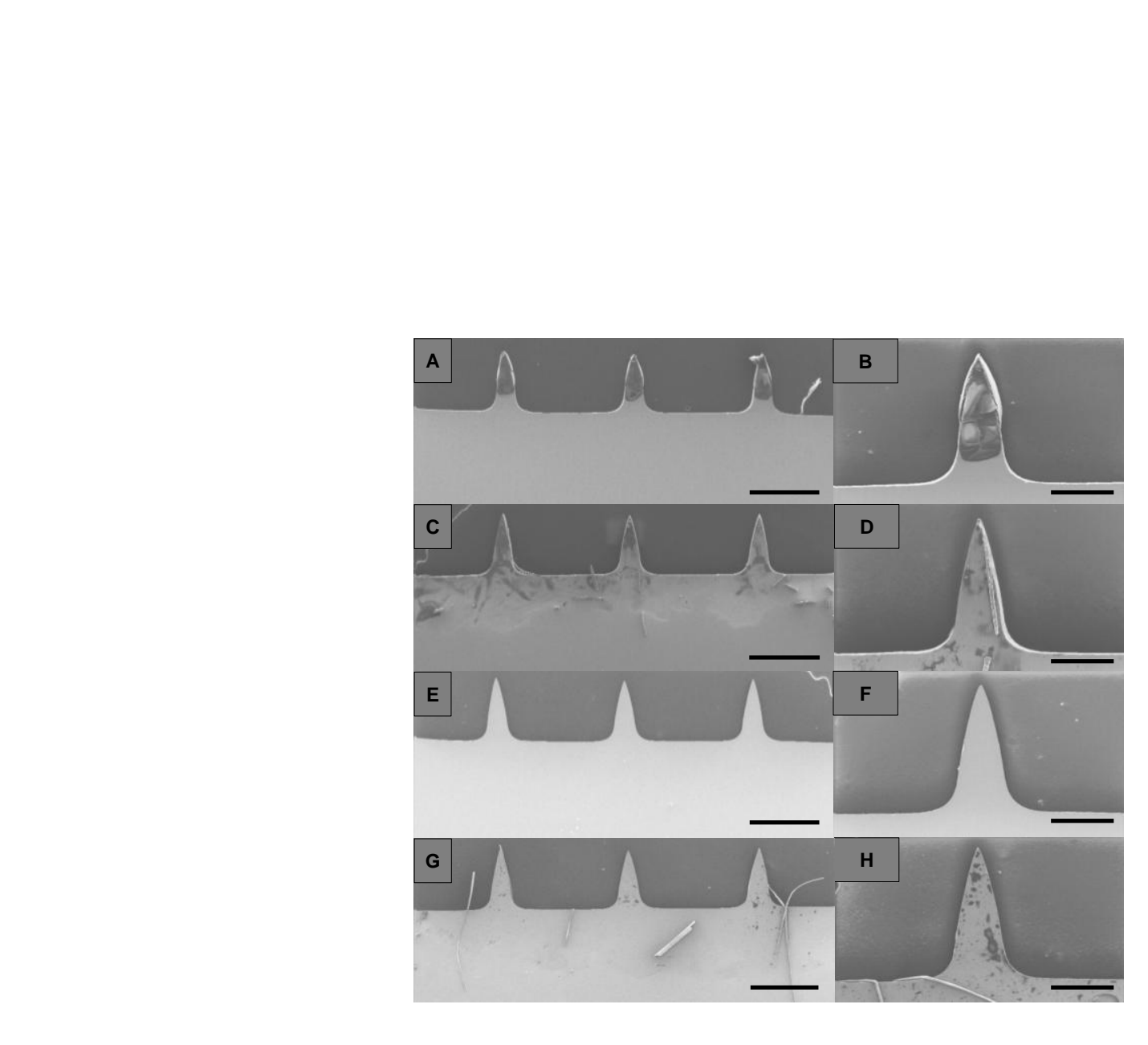

Fig.5.

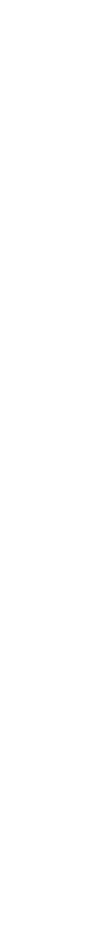

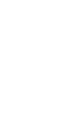
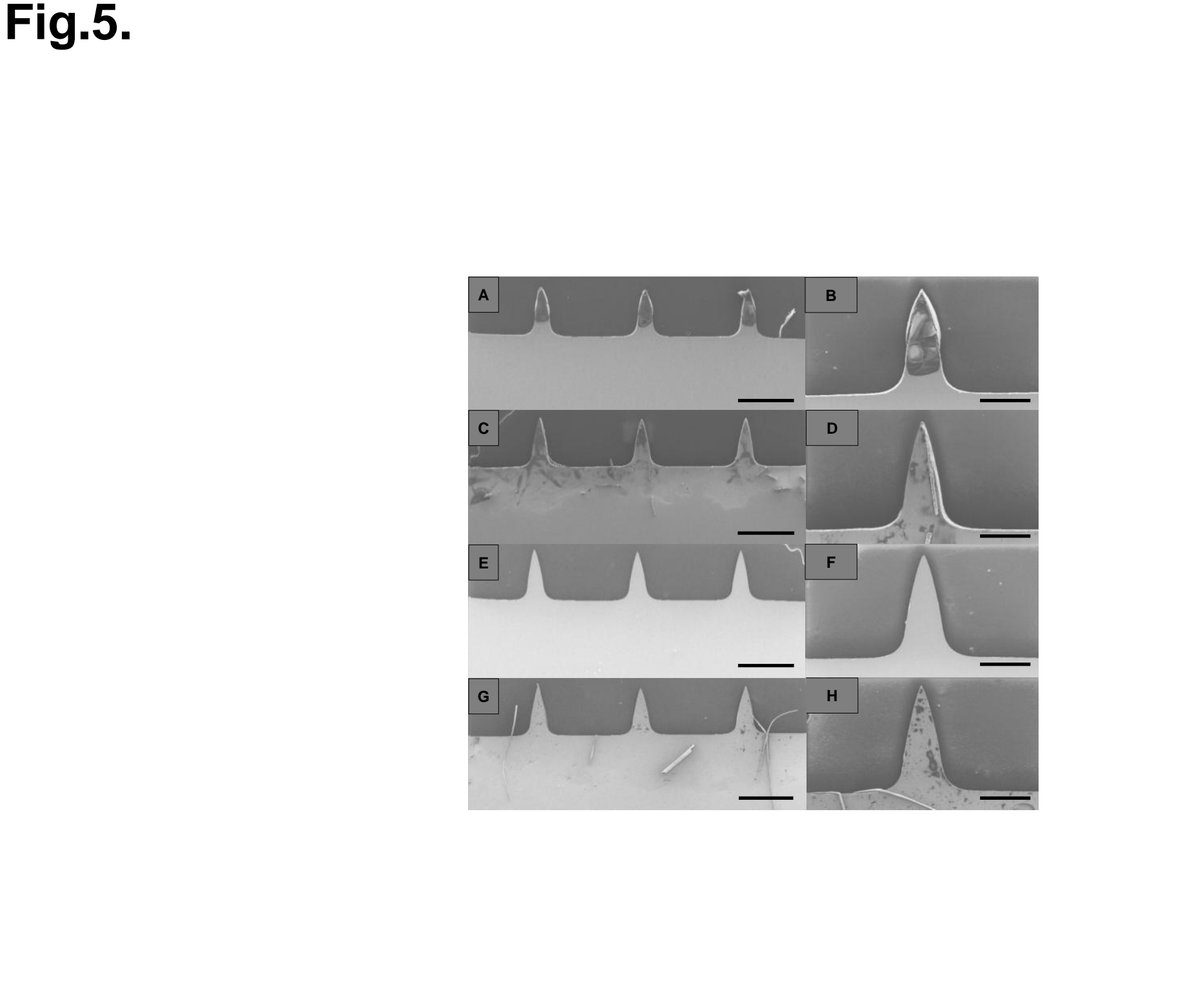
Fig.6.

A

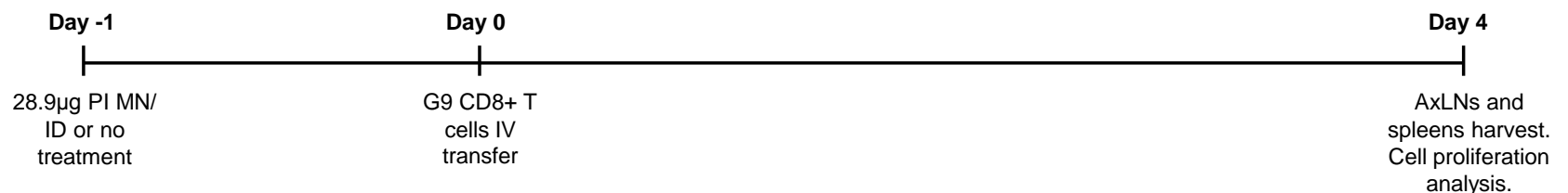

B

Control

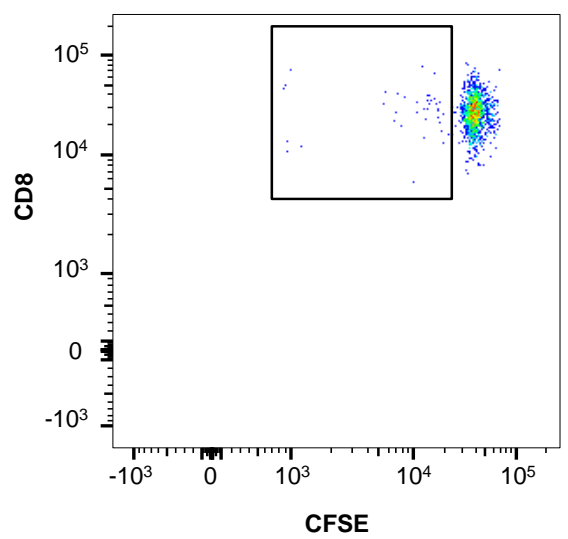

C
MN

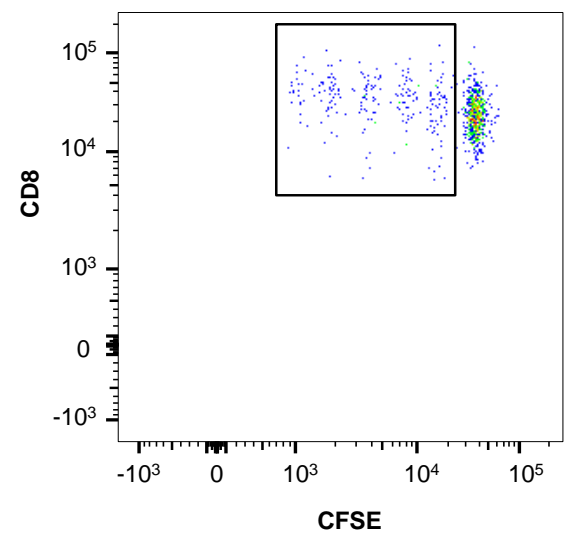

ID

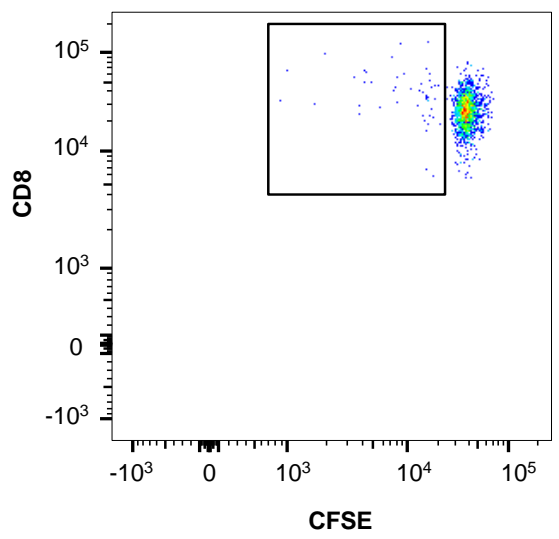

ns

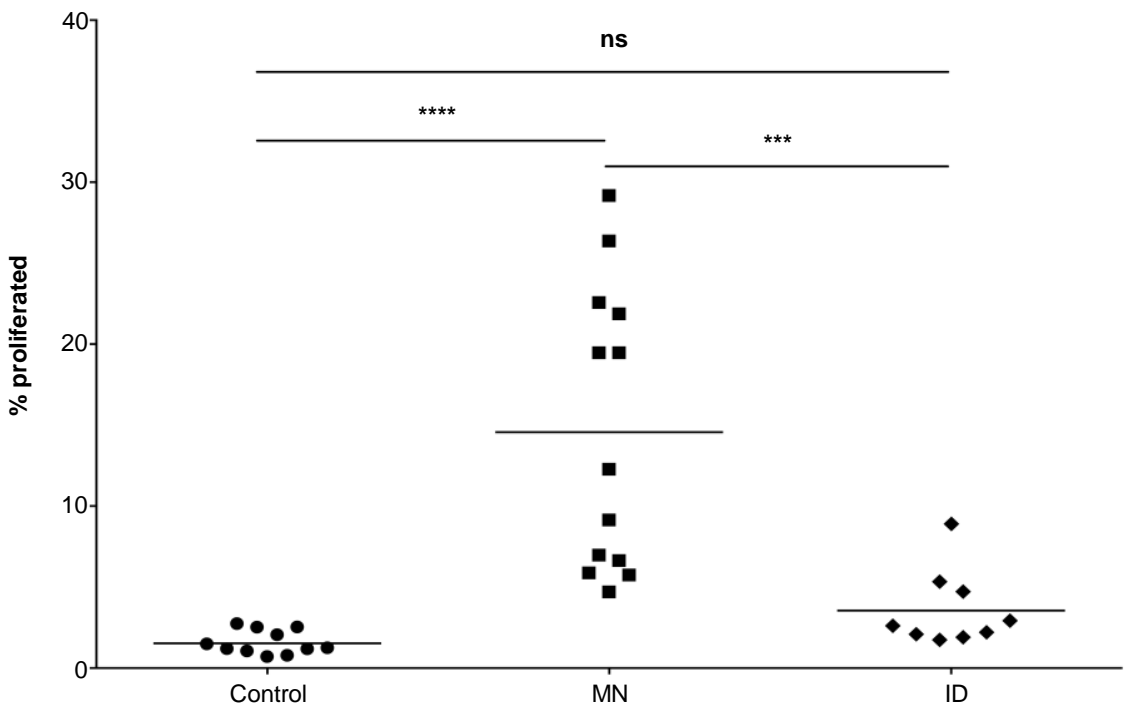

Control 


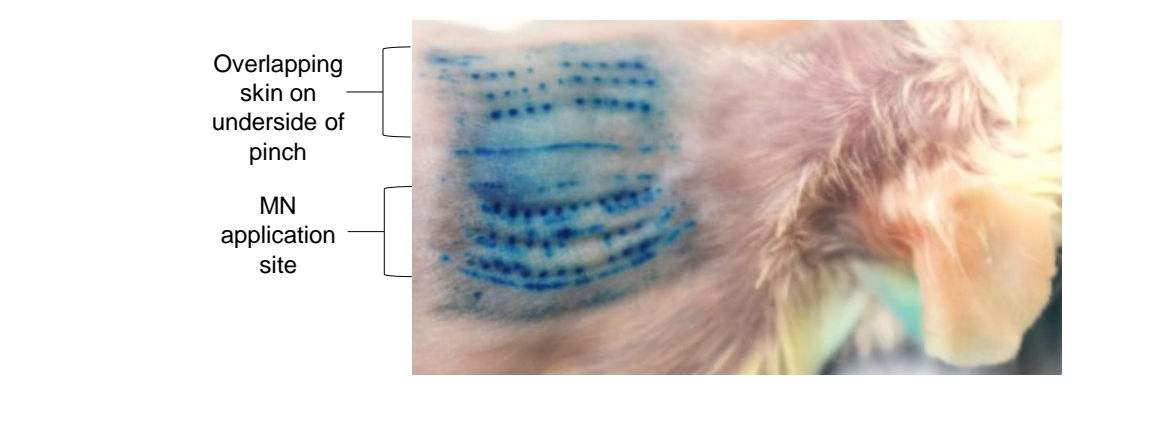

Figure(s) 3.

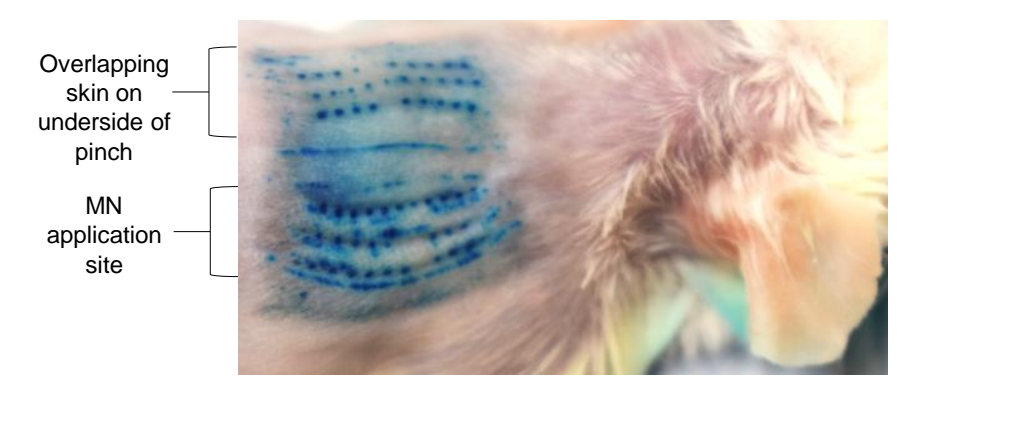

Fistgr(s).
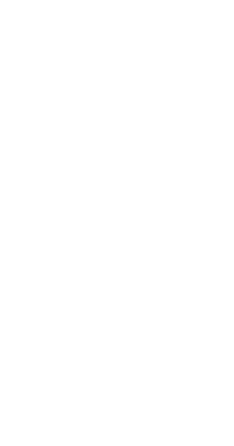
(1) (1) (1) (1)

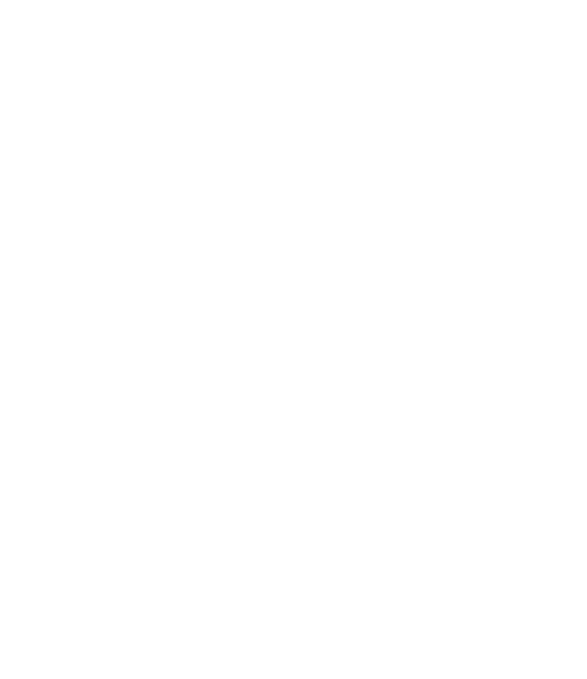





\section{CRediT author statement}

Farah Arikat: Methodology, Validation, Formal analysis, Investigation, Data curation, Writing-Original Draft, Visualization, Project administration. Stephanie J. Hanna: Conceptualization, Methodology, Validation, Formal analysis, Investigation, Writing-Review \& Editing, Supervision, Project administration, Funding acquisition. Ravinder K. Singh: Methodology, Formal analysis, Investigation, Writing-Review \& Editing. Luciano Vilela: Resources, Writing-Review \& Editing. F. Susan Wong: Conceptualization, Methodology, Validation, Resources, Writing-Review \& Editing, Supervision, Project administration, Funding acquisition. Colin M. Dayan: Conceptualization, Methodology, Validation, Resources, Writing-Review \& Editing, Supervision, Project administration, Funding acquisition. Sion A. Coulman: Conceptualization, Methodology, Validation, Resources, Writing-Original Draft, Writing-Review \& Editing, Supervision, Project administration, Funding acquisition. James C. Birchall: Conceptualization, Methodology, Validation, Resources, Writing-Review \& Editing, Supervision, Project administration, Funding acquisition. 


\section{Highlights:}

- Proinsulin is a multi-epitope immunotherapy candidate for type 1 diabetes.

- Steel microneedle arrays were uniformly coated with $>30 \mu \mathrm{g}$ of proinsulin.

- A high proportion (86\%) of coated proinsulin was delivered to the local tissue.

- Antigen-specific T cells proliferated in local lymph nodes in response.

- Microneedle delivery resulted in greater proliferation than intradermal injection. 